\title{
The large time asymptotics of the entropy
}

\author{
Lei $\mathrm{Ni}$
}

May 5, 2009

In this note, due to several requests after the the publication of [N2], as well as new potential applications emerging, we supply the details of the computation on the asymptotics of the entropy stated in [N1], Corollary 4.3, and Proposition 1.1 of [N2]. It is my pleasure to contribute this paper to the birthday of Professor Rothschild.

In this note we assume that $(M, g)$ is a complete Riemannian manifold of dimension $n$ with nonnegative Ricci curvature. We also further assume that it has maximum volume growth. Namely

$$
\lim _{r \rightarrow \infty} \frac{V_{x}(r)}{r^{n}}>0
$$

where $V_{x}(r)$ is the volume of the ball of radius $r$ centered at $x$. Let $H(x, y, t)$ be the heat kernel, or equivalently the minimum positive fundamental solution to the heat operator $\frac{\partial}{\partial t}-\Delta$. For this note we fix $x$ and write simply abbreviate $H(x, y, t)$ as $H(y, t)$, and $V_{x}(r)$ as $V(r)$. Also we simply denote the distance between $x$ and $y$ by $r(y)$. Recall the definition of the Perelman's entropy

$$
\mathcal{W}(H, t):=\int_{M}\left(t|\nabla f|^{2}+f-n\right) H d \mu
$$

where $f=-\log H-\frac{n}{2} \log (4 \pi t)$. Also recall the Nash entropy

$$
\mathcal{N}(H, t)=-\int_{M} H \log H d \mu-\frac{n}{2} \log (4 \pi t)-\frac{n}{2} .
$$

Let $\nu_{\infty}$ be the cone angle at infinity which can be defined by

$$
\nu_{\infty}:=\lim _{r \rightarrow \infty} \frac{\theta(r)}{\omega_{n}}
$$

where $\theta(r):=\frac{V(r)}{r^{n}}, V(r)$ is the volume of the ball $B_{x}(r)$ centered at $x$ and $\omega_{n}$ is the volume of the unit ball in $\mathbb{R}^{n}$.

Recall that in [N2], page 331, there we have proved that

$$
\lim _{t \rightarrow \infty} \mathcal{W}(H, t)=\lim _{t \rightarrow \infty} \mathcal{N}(H, t) .
$$

Moreover, it was also proved in [N1] that $M$ is of maximum volume growth if and only if $\lim _{t \rightarrow \infty} \mathcal{W}(H, t)>-\infty$. The main purpose here is to supply the detail of the following claim. 
Theorem 1 For any $x \in M$,

$$
\lim _{t \rightarrow \infty} \mathcal{W}(H, t)=\lim _{t \rightarrow \infty} \mathcal{N}(H, t)=\log \nu_{\infty}
$$

Besides that it is interesting to be able to detect the geometric information such as the volume ratio $\nu_{\infty}$ from the large time behavior of the entropy, there appeared an extra indication of possible uses of such a result. In an very interesting paper [Ka], Kaimanovich defined an entropy $h(\widetilde{M})$ on the space of so-called minimal Martin boundary of the positive harmonic functions on the universal/a regular covering space $\widetilde{M}$ of a compact Riemannian manifold. More precisely, let $\mathcal{H}(\widetilde{M})$ be the vector space with the seminorms $\|u\|_{K}=\sup _{K}|u|$, where $K$ is a compact subset. Let $\mathcal{K}_{p}=\{u \mid u \in \mathcal{H}(\widetilde{M}), u(p)=1, u>0\}$. This is a convex compact subset. Define the minimal Martin boundary of $\widetilde{M}$ by $\partial^{*} \widetilde{M}=\left\{u \in \mathcal{K}_{p}\right.$, and $u$ minimal $\}$. Here $u>0$ is called minimal if for any nonnegative harmonic function $h \leq u, h$ must be a multiple of $u$. This immediately implies the representation formula: for any positive harmonic function $f$, there exists a Borel measure $\mu^{f}$ on $\partial^{*} \widetilde{M}$ such that

$$
f(x)=\int_{\partial^{*} \widetilde{M}} u(x) d \mu^{f}(u) .
$$

In particular, there exists a measure $\nu$ corresponding to $f \equiv 1$. Now $u(x) d \nu(u)$ is also a probability measure. The points $x \in \widetilde{M}$ can be identified with the probability measure $u(x) d \nu(u)$ on $\partial^{*} \widetilde{M}$. The so-called relative entropy is defined to be $\phi(x, y)=-\int_{\partial^{*} \widetilde{M}} \log \left(\frac{u(x)}{u(y)}\right) u(y) d \nu(u)$. By Jensen's inequality it is easy to see that $\phi(x, y) \geq 0$. The Kaimanovich's entropy is defined by averaging $\phi(x, y)$ as following: First check that

$$
\frac{1}{\tau} \int_{\widetilde{M}} \phi(x, y) H(\tau, x, y) d \mu_{\widetilde{M}}(y)
$$

is a function independent of $\tau$ and also descends to $M$. Here $H(\tau, x, y)$ is the heat kernel of $\widetilde{M}$. Then define

$$
h(\widetilde{M}) \doteqdot \int_{M}\left(\frac{1}{\tau} \int_{\widetilde{M}} \phi(x, y) H(\tau, x, y) d \mu_{\widetilde{M}}(y)\right) d \mu_{M}(x) .
$$

Here we normalize so that $\mu_{M}(x)$ is a probability measure on $M$. From the above definition it was proved by Kaimanovich [Ka] (see also [W]) that

$$
h(\widetilde{M})=\int_{M} \int_{\widetilde{M}} u(x)|\nabla \log u(x)|^{2} d \nu(u) d \mu(x) .
$$

Hence the positivity of $h(\widetilde{M})$ implies the existence (in fact ampleness) of nonconstant positive harmonic functions on $\widetilde{M}$. Most interestingly, the Theorem 2 of [Ka] asserts that

$$
h(\widetilde{M})=-\lim _{t \rightarrow \infty} \frac{1}{t} \int_{\widetilde{M}} H(t, x, y) \log H(t, x, y) d \mu(y)
$$

for any $x$. This is sensational since it is not hard to check for instance that when $\lambda(\widetilde{M})$, the bottom of the $L^{2}$-spectrum of the Laplace operator on $\widetilde{M}$ is positive, $h(\widetilde{M}) \geq 4 \lambda(\widetilde{M})[\mathrm{L}]$. In fact the above result of Kaimanovich plays a crucial role in [W], where Xiaodong Wang solves 
a conjecture of Jiaping Wang on characterizing the universal cover of a compact Riemannian manifolds being the hyperbolic space by the information on $\lambda(\widetilde{M})$ and the lower bound of Ricci curvature of $M$. (See also [Mu] for the related work on Kähler manifolds.) Motivated by the above discussions, mainly the entropy of Kaimanovich [Ka] and its above mentioned connection with the large time behaviors of the heat kernel and its direct implications on the existence of harmonic functions, we propose the following problem.

Problem 1 Let $M$ be a complete Riemannian manifold of positive sectional curvature. When does $M$ admit non-constant harmonic functions of polynomial growth?

Here a harmonic function $u(x)$ is of polynomial growth if there exist positive constants $d, C_{u}$ such that

$$
|u(y)| \leq C_{u}(1+r(y))^{d} .
$$

We conjecture that the necessary and sufficient condition is that $\lim _{t \rightarrow \infty} \mathcal{N}(H, t)>-\infty$, namely $M$ is of maximum volume growth. Note that for the corresponding problem on holomorphic functions of polynomial growth, the sufficient part has been solved by the author provided that the manifold is Kähler with bounded non-negative bisectional curvature (cf.[N4]).

Now we devote the rest of the paper to the detailed proof of Theorem 1. Under the above notation, let us first recall a result of Li, Tam and Wang [LTW]. A computation similar as below appeared in the earlier paper [N3], pages 935-936.

Theorem 2 [Li-Tam-Wang] Let $\left(\mathcal{M}^{n}, g\right)$ be a complete Riemannian manifold with nonnegative Ricci curvature and maximum volume growth. For any $\delta>0$, the heat kernel of $\left(\mathcal{M}^{n}, g\right)$ satisfies

$$
\begin{aligned}
& \frac{\omega_{n}}{\theta(\delta r(y))}(4 \pi t)^{-\frac{n}{2}} \exp \left(-\frac{1+9 \delta}{4 t} r^{2}(y)\right) \leq H(y, t) \\
& \quad \leq\left(1+C\left(n, \theta_{\infty}\right)(\delta+\beta)\right) \frac{\omega_{n}}{\theta_{\infty}}(4 \pi t)^{-\frac{n}{2}} \exp \left(-\frac{1-\delta}{4 t} r^{2}(y)\right),
\end{aligned}
$$

where $\theta_{\infty}=\lim _{r \rightarrow \infty} \theta(r)$,

$$
\beta:=\delta^{-2 n} \max _{r \geq(1-\delta) r(y)}\left(1-\frac{\theta_{x}(r)}{\theta_{x}\left(\delta^{2 n+1} r\right)}\right) .
$$

Note that $\beta$ is a function of $r(y)$ and

$$
\lim _{r(y) \rightarrow \infty} \beta=0 .
$$

Therefore, for any $\epsilon>0$, there exists a $B$ sufficiently large such that if $r(y) \geq B$ we have

$$
\begin{aligned}
& \frac{\omega_{n}}{\theta_{\infty}}(1-\epsilon)(4 \pi t)^{-\frac{n}{2}} \exp \left(-\frac{1+9 \delta}{4 t} r^{2}(y)\right) \leq H(y, t) \\
& \quad \leq\left(1+C\left(n, \theta_{\infty}\right)(\delta+\epsilon)\right) \frac{\omega_{n}}{\theta_{\infty}}(4 \pi t)^{-\frac{n}{2}} \exp \left(-\frac{1-\delta}{4 t} r^{2}(y)\right) .
\end{aligned}
$$

We can also require that $\theta\left(\delta^{2 n+1} r\right) \leq(1+\epsilon) \theta_{\infty}$. 
The upper estimates: First by the lower estimate of Li-Tam-Wang,

$$
\begin{aligned}
\mathcal{N}(H, t) & \leq-\int_{M} H \log \left(\frac{\omega_{n}}{\theta(\delta r(y))}\right) d \mu+\int_{M} H\left(\frac{1+9 \delta}{4 t} r^{2}(y)\right) d \mu-\frac{n}{2} \\
& =I+I I-\frac{n}{2} .
\end{aligned}
$$

We shall estimate $I$ and $I I$ below as in [N3]. Split

$$
I=-\int_{0}^{B}-\int_{B}^{\infty}\left(\int_{\partial B(s)} H \log \left(\frac{\omega_{n}}{\theta(\delta r(y))}\right) d A\right) d s=I_{1}+I_{2} .
$$

It is easy to see that

$$
\lim _{t \rightarrow \infty} I_{1} \leq 0 .
$$

To compute $I I_{2}$ we now make use of the lower estimate in (1) to have that

$$
\begin{aligned}
I_{2} & \leq-(1-\epsilon) \frac{\omega_{n}}{\theta_{\infty}}(4 \pi t)^{-\frac{n}{2}} \int_{B}^{\infty} \int_{\partial B(s)} \exp \left(-\frac{1+9 \delta}{4 t} s^{2}\right) \log \left(\frac{\omega_{n}}{\theta(\delta s)}\right) d A d s \\
& \leq \log \left(\frac{(1+\epsilon) \theta_{\infty}}{\omega_{n}}\right)(1-\epsilon) n \omega_{n}(4 \pi t)^{-\frac{n}{2}} \int_{B}^{\infty} \exp \left(-\frac{1+9 \delta}{4 t} s^{2}\right) s^{n-1} d s .
\end{aligned}
$$

Here we have used that $\theta(\delta r(y)) \leq \theta_{\infty}(1+\epsilon)$ and the surface area of $\partial B(s)$ satisfies $A(s) \geq$ $n \theta_{\infty} s^{n-1}$. Computing the integral via the change of variable $\tau=\frac{1+9 \delta}{4 t} s^{2}$ and taking $t \rightarrow \infty$ we have that

$$
\lim _{t \rightarrow \infty} I_{2} \leq \log \left(\frac{(1+\epsilon) \theta_{\infty}}{\omega_{n}}\right)(1-\epsilon)(1+9 \delta)^{-n / 2} .
$$

The estimate of $I I$ is very similar. Using the Gamma function identity

$$
\Gamma\left(\frac{n}{2}+1\right)=\Gamma\left(\frac{n}{2}\right) \frac{n}{2}
$$

we can have that

$$
\lim _{t \rightarrow \infty} I I \leq(1+\epsilon)\left(1+C\left(n, \theta_{\infty}\right)(\delta+\epsilon)\right)(1+9 \delta)(1-\delta)^{n / 2+1} \frac{n}{2} .
$$

Summarizing we have that

$$
\begin{aligned}
\lim _{t \rightarrow \infty} \mathcal{N}(H, t) \leq & \log \left(\frac{(1+\epsilon) \theta_{\infty}}{\omega_{n}}\right)(1-\epsilon)(1+9 \delta)^{-n / 2} \\
& +(1+\epsilon)\left(1+C\left(n, \theta_{\infty}\right)(\delta+\epsilon)\right)(1+9 \delta)(1-\delta)^{n / 2+1} \frac{n}{2}-\frac{n}{2}
\end{aligned}
$$

Letting $\epsilon \rightarrow 0$, then $\delta \rightarrow 0$ we have what

$$
\lim _{t \rightarrow \infty} \mathcal{N}(H, t) \leq \log \nu_{\infty}
$$

For the lower estimate, we use the other inequality provided by Theorem 2, mainly (1). First write

$$
\begin{aligned}
\mathcal{N}(H, t) & \geq \int_{M} H\left(\frac{1-\delta}{4 t}\right) r^{2}(y) d \mu-\log \left[\left(1+C\left(n, \theta_{\infty}\right)(\delta+\epsilon)\right) \frac{\omega_{n}}{\theta_{\infty}}\right]-\frac{n}{2} \\
& =\int_{r \leq B}+\int_{r \geq B} H\left(\frac{1-\delta}{4 t}\right) r^{2}(y) d \mu-\log \left[\left(1+C\left(n, \theta_{\infty}\right)(\delta+\epsilon)\right) \frac{\omega_{n}}{\theta_{\infty}}\right]-\frac{n}{2}
\end{aligned}
$$


Similarly

$$
\lim _{t \rightarrow \infty} \int_{r \leq B} H\left(\frac{1-\delta}{4 t}\right) r^{2}(y) d \mu \rightarrow 0
$$

as $t \rightarrow \infty$. On the other hand, using that $A(r) \geq n \theta_{\infty} r^{n-1}$,

$$
\begin{aligned}
\lim _{t \rightarrow \infty} I_{3} & \doteqdot \int_{r \geq B} H\left(\frac{1-\delta}{4 t}\right) r^{2}(y) d \mu \\
& \geq \lim _{t \rightarrow \infty} n \omega_{n}(1-\epsilon)(1-\delta) \frac{1}{(4 \pi t)^{n / 2}} \int_{B}^{\infty} \exp \left(-\frac{(1+9 \delta) r^{2}}{4 t}\right) \frac{r^{2}}{4 t} r^{n-1} d r .
\end{aligned}
$$

The direct calculation shows that

$$
\lim _{t \rightarrow \infty} \frac{1}{(4 \pi t)^{n / 2}} \int_{B}^{\infty} \exp \left(-\frac{(1+9 \delta) r^{2}}{4 t}\right) \frac{r^{2}}{4 t} r^{n-1} d r=\frac{1}{2} \Gamma\left(\frac{n}{2}+1\right)(1+9 \delta)^{-\frac{n}{2}-1} .
$$

Using $\omega_{n}=\frac{2 \pi^{n / 2}}{\Gamma\left(\frac{n}{2}\right) n}=\frac{\pi^{n / 2}}{\Gamma\left(\frac{n}{2}+1\right)}$, we finally have that

$$
\lim _{t \rightarrow \infty} I_{3} \geq \frac{n}{2}(1-\epsilon)(1-\delta)(1+9 \delta)^{-\frac{n}{2}-1} .
$$

The lower estimate

$$
\lim _{t \rightarrow \infty} \mathcal{N}(H, t) \geq \log \nu_{\infty}
$$

follows after we taking $\epsilon \rightarrow 0$ and then $\delta \rightarrow 0$.

\section{References}

[Ka] V. A. Kaimanovich, Brownian motion and harmonic functions on covering manifolds. An entropy approach. Soviet Math. Dokl. 33 (1986), 812-816.

[L] F. Ledrappier, Harmonic measures and Bowen-Margulis measures. Isreal J. Math. 71(1990), 275-287.

[LTW] P. Li, L.-F. Tam and J. Wang, Sharp bounds for the Green's function and the heat kernel, Math. Res. Lett. 4 (1997), no. 4, 589-602.

[Mu] Munteanu, $\mathrm{O}$ On a characterization of the complex hyperbolic space. ArXiv:0802.0307.

[N1] L. Ni, The entropy formula for linear heat equation, J. Geom. Anal. 14(2004), 87100 .

[N2] L. Ni, Addenda to "The entropy formula for linear heat equation", J. Geom. Anal. 14(2004), 369-374.

[N3] L. Ni, A monotonicity formula on complete Kaehler manifolds with nonnegative bisectional curvature, J. Amer. Math. Soc. 17 (2004), 909-946.

[N4] L. Ni, Ancient solutions to Kähler-Ricci flow. Math. Res. Lett. 12 (2005), no. 5-6, 633-653. 
[W] X.-D. Wang, Harmonic functions, entropy, and a characterization of the hyperbolic space, preprint.

\author{
AdDRESSES \\ LEI NI, Department of Mathematics, University of California at San Diego, La Jolla, CA
} 92093, USA

email: lni@math.ucsd.edu 